\title{
Obesity Defined as Excess Storage of Inert Triglycerides - Do We Need a Paradigm Shift?
}

\author{
Thorkild I.A. Sørensen \\ Institute of Preventive Medicine, Copenhagen University Hospitals, Capital Region of Denmark, Denmark
}

Obesity affects three domains of individual life: the physical effects of the body size and weight, the psychosocial effects of the abnormal body image and the various metabolic effects predisposing to a broad panel of co-morbidities. In this editorial, I will argue for the need of a fundamental revision of the concept of obesity as a health problem primarily in the latter domain. The argument is based on the accumulating evidence that the stored triacylglyceride (TAG) is an inert biochemical compound making the body fat an innocent bystander [1]. The metabolic health problems appear avoidable as long as the TAG stores can be expanded and emerge only when the limits are reached $[2,3]$. This argument may elicit a need for new lines of research that can provide the basis for another definition of the obesity problem and hence for novel strategies for prevention and management of the condition and its associated health problems. Owing to the various ways of relationships between the three domains, a new definition based on the metabolic problems may have implications also for the two other domains.

While acknowledging that there is a very well established association between accumulation of TAG in various body compartments and the metabolic health effects [4, 5], there are reasons to dissociate these observations from an interpretation of a causal relationship between TAG accumulation and the health effects. The immediate major reason to challenge the conventional idea of the impact of the amount of the accumulated TAG is the fact that there are individual deviations from the association with the metabolic alterations and with the risk of the clinical problems, i.e. the deviations that make the correlations less than 1.0. Although such departures are still compatible with a causal role of TAG, the complete disconnections in extreme scenarios raise serious doubts. The classical epidemiological problem of unknown confounding may apply here; the association of TAG accumulation with metabolic alterations may be due to other factors inducing these alterations and co-occurring with the TAG accumulation.

\section{KARGER \\ Fax +497614520714 \\ Information@Karger.de}

www.karger.com (c) 2011 S. Karger GmbH, Freiburg

$1662-4025 / 11 / 0042-0091 \$ 38.00 / 0$

Accessible online at:

www.karger.com/ofa
The current definition of obesity is based on the assumed adverse health effects of excessive amounts of body fat relative to some arbitrary standard assumed to convey the lowest risk. Particular emphasis has been put on the association with the risk of hard clinical endpoints such as diabetes, cardioand cerebrovascular diseases, cancer and mortality. Longterm, very large-scale field studies are needed to get adequate statistical power for estimation of the risk functions $[4,5]$. Because of the technical requirements in measuring total body fat mass, too few studies provide sufficient evidence for the quantitative relationships between amount of body fat and the adverse health effects $[6,7]$. Therefore, the definition has been translated to a definition based on body weight, which, when adjusted for the influence of lean mass indicated by height as in BMI (i.e. weight/height ${ }^{2}$ ), exhibits a high correlation with the amount of body fat. This has led to a generally accepted definition of obesity as BMI of at least $30 \mathrm{~kg} / \mathrm{m}^{2}$.

However, many, if not the majority, of individuals with a BMI above 30 in any one population do not suffer from the adverse health effects. Although starting adult life as obese does reduce life expectation, many of them survive until old age [8]. Women carry more TAG than men but do not have greater risks of adverse health effects. The large Asian populations are suffering from a severe diabetes epidemic even though they are still less obese than the Western populations, probably also when taking into account their relatively greater amount of body fat at similar BMI. As the most extreme case, around $10-20 \%$ of the massively obese patients, assessed in preparation for bariatric surgery, do not suffer from the metabolic alterations or signs of the related clinical problems, an observation that has led to the prevailing distinction between the healthy and unhealthy obese patients. In the opposite situation, patients who for various reasons suffer from lipodystrophy, where the usual fat deposition in adipose tissue is compromised, may have severe metabolic alterations and consequently a high risk of the associated clinical problems. These observations combined suggest that the adverse health effects 
may arise when limits of TAG storage have been reached and that these limits may differ between individuals as well as between population groups.

The distribution of fat in the body, manifested in different body shapes, is associated with the metabolic health risk independently of the total amount of body fat [5]; the android, truncal or abdominal form of obesity appears more dangerous than the gynoid, peripheral or gluteofemoral form. Inclusion of body fat distribution is considered a qualification of the definition of obesity. Techniques for objective measurement of body fat distribution, needed in field studies for estimation of the association with the major diseases, has not been available. Waist and hip circumferences have been used as proxy measure of the body fat distribution, and proposals have been made for definitions based on these measures. In order to disentangle the influence of the body shape from that of body weight, it has been proposed to use the ratios between the circumferences or to use the circumferences adjusted for BMI or for height. Waist circumference appears to capture a major part of the health risks associated with amount of body fat [9].

As for total body fat and its proxy measure, BMI, many individuals with the more risky body fat distribution do not suffer from the adverse health effects, suggesting that the mere accumulation of TAG in these depots is not the reason for the health problems. On the other hand, the risk is particularly elevated when abdominal deposition is combined with little general body fat mass. This leads to the hypothesis that the abdominal accumulation is a consequence of limitations of the TAG storage capacity in the larger peripheral fat depots, and that the associated health problems reflect that also limitations in the abdominal fat depots have been reached.

More recently, it has been recognized that the fat deposition in ectopic sites, which means accumulation of TAG in other tissues than adipose tissue, for example in the liver and in skeletal muscles, are associated with particular high risk of the metabolic alterations (insulin resistance, glucose intolerance, dyslipidemia) that may lead to the increased risk of the diseases considered as the main contributors to the metabolic health problems of obesity. There is a correlation between the amount of ectopic TAG accumulation and the degree of the metabolic alterations [10]. The likelihood of carrying ectopic TAG accumulation increases by degree of overall obesity and especially by degree of abdominal obesity [10,11]. On the other hand, patients with lipodystrophy may develop very severe ectopic fat accumulation. The ectopic fat storage has been considered as the main reason for the metabolic alterations and the subsequent clinical problems in obesity.

However, even for the ectopic fat accumulation, the moderate level of the correlation suggests that a considerable proportion of these individuals do not suffer from the adverse health effects, evidenced by the metabolic alterations. That the mere ectopic TAG accumulation as such may not lead to these problems is convincingly indicated by the recently identified variant in the adiponutrin gene, PNPLA3, which is as- sociated with an increased accumulation of TAG in the liver, but also by less metabolic alterations than in individuals without this gene variant [12]. Thus, the ectopic TAG accumulation may not be the cause of the adverse metabolic health effects. In agreement with the hypothesis about limitations in TAG storage in both peripheral and abdominal depots, the association between ectopic fat accumulation and adverse metabolic effects may reflect limitations in the ability to store TAG ectopically.

The theory of the relations between adipose tissue expandability and metabolic alterations has been thoroughly investigated in rodent models, combining various specific genetic modifications with dietary manipulations $[2,3]$. These studies have served to test specific hypotheses derived from the theory and have in general consolidated the theory. As the most extreme scenario, such experiments have shown that blocking the formation of TAG by prevention of the binding of the third fatty acid to diglycerides is associated with severe metabolic alterations.

The disconnection between TAG accumulation, whether occurring in adipose tissue or ectopically, and the metabolic alterations strongly suggest that TAG in itself is an inert compound when deposited as intracellular TAG droplets. TAG accumulation is essentially a beneficial biological process, primarily serving as storage of energy reserves. The evidence suggests that there are limits to how much TAG can be accumulated at various sites, and that these limits may show considerable individual variation, ranging from the severely affected lipodystrophic patients through the metabolically healthy, but massively obese patients. The metabolic alterations apparently emerge when these limits are exceeded. The abdominal and ectopic deposition of TAG may be seen as a stepwise adaptation to the exceeded limits for further fat deposition in the peripheral or abdominal adipose tissue. As demonstrated by the lipodystrophic patients and the animal models, the occurrence of TAG accumulation apparently reduces the risk of the adverse metabolic effects compared to alternative scenarios where the TAG accumulation is inhibited; so, under these conditions the ability to store TAG may even be considered as a protective mechanism.

This interpretation of the TAG accumulation has several important implications for the research aiming at understanding and managing the metabolic alterations and the subsequent health problems.

First, it makes little sense to measure how much TAG has been accumulated, whether in adipose tissue or at ectopic sites, such as the liver and skeletal muscles, unless this is compared to how much TAG can be stored by the same individual. The amount of accumulated TAG can be considered an innocent bystander, an epiphenomenon, of little relevance by itself for the abnormal metabolism.

Second, it is worthwhile to investigate the limits of TAG accumulation. Are the limits analogous to thresholds, or are they reflecting a continuous progressive process? How is the 
structure and function of the adipose tissue altered when the limits are exceeded? Does a maximum of adipocyte size or other aspects of the TAG storage process define the limits as suggested in the theory about the hypertrophic versus hyperplastic type of obesity? How can the limits be identified before they are reached? What are the determinants of the limits, and which of these determinants are responsible for the individual variation? Can the individual limits change over time, and can they be manipulated?

Third, the health perspectives of the interest in the causes and processes of TAG accumulation [13, 14], including areas of regulation of energy and macronutrient balance, appetite and food intake, physical activity and energy expenditure, formation of adipose tissue, and adipocyte fat storage, need to be put in the double frame of being potentially beneficial until turning into health problems when the limits of TAG storage have been exceeded.

Fourth, the processes that may lead to the overloading of the capacity for TAG accumulation should be further investigated. Is this just a consequence of intake of too much fat relatively to the needs of fat as energy source? Obviously, the need for storage of TAG is dependent on how much is oxidized relative to how much is consumed. Is excess consumption of carbohydrates replacing the fat as energy source a main determinant? Is increased oxidation of fatty acids by physical activity important as a tool to avoid the overloading? Is it possible to avoid exceeding the limits by increasing oxidation of fat in the adipose tissue (enhance processes similar to those predominating in brown adipose tissue)?

Fifth, understanding what is inducing the metabolic alterations and subsequent health problems when the limits are exceeded is of paramount importance. It is likely that several mechanisms are involved as recently extensively reviewed [15]. A toxic effect of excess free fatty acids may be a central element as implicated in the so-called 'lipotoxicity' theory. An increased secretion from the adipocytes of insulin resistance-inducing adipokines (tumor necrosis factor alpha, interleukin-6, and others) may be early consequences of the exceeded limits of TAG accumulation. Endoplasmatic reticulum stress, mitochrondrial dysfunction, reactive oxygen species activation, and initiation of inflammatory processes may be core elements in the pathogenic development leading to insulin resistance and subsequent metabolic alterations. Identification of pathways allowing inhibition or abating the consequences of the metabolic alterations and health problems in due time become major tasks. It may be expected that interference with the process of exceeding the limits of TAG storage is better than awaiting the emergence of the metabolic disturbances, for example insulin resistance or later dysfunctions manifest in the metabolic syndrome.
Sixth, research aiming at development of improved preventive strategies should be targeted at the avoidance of exceeding the limits of TAG storage and therapeutic strategies should be targeted at reducing the adverse effects of exceeding these limits. Prevention of weight gain and weight loss may not necessarily be the right strategy by itself unless the procedures used also solve the problems defined by the limits of TAG storage. The finding that thiazolidinediones reduce the risk of development of diabetes and at the same time increase the TAG storage illustrates the need to specify the target [16]. The excess mortality associated with weight loss, observed in a number of large-scale prospective population studies, may also show the need to target the weight loss to the individuals in whom the TAG storage has been exceeded; effects of weight loss in individuals who have not reached this limit may be dominated by complications due to loosing tissue mass also outside the adipose tissue [17]. The great challenge is to develop tools to measure where the individual is at a given point in time in relation to his or her own limits for TAG storage and to introduce this as a basis for indications to intervene.

Seventh, the obesity epidemic, showing up in the increasing prevalence of obesity, defined by BMI above $30 \mathrm{~kg} / \mathrm{m}^{2}$, may need to be conceived as an adaptation at the population level to the challenges of the capacity to store TAG. In a contrafactual scenario, where the obesity epidemic had not developed because of low capacity to store TAG, the adverse metabolic health consequences might have been much worse in the Western world and perhaps similar to the current dramatic and devastating development of the diabetes epidemic and all what follows it in the Asian populations.

In conclusion, the recognition that TAG accumulation is a harmless and perhaps even beneficial biological process implies that definition of obesity on the basis of this feature needs to be replaced by a definition that focus on the risk of exceeding the individual limits for continued TAG accumulation. It may be considered a paradigm shift with several profound implications for research in obesity aimed at assessment, characterization, causes, consequences, mechanisms and development of novel targets for prevention and treatment.

\section{Acknowledgment}

I thank Professor Antonio Vidal-Puig, Cambridge University, and other colleagues in the integrated project, 'Hepatic and adipose tissue and functions in the metabolic syndrome' (HEPADIP, see www.hepadip.org) under the European 6th Framework, for introducing me to the lipotoxicity theory and encouraging me to consider its clinical and epidemiological implications. The work is part of the Danish Obesity Research Center (DanORC, see www.danorc.dk). 


\section{References}

1 Sørensen TIA, Virtue A, Vidal-Puig A: Obesity as a clinical and public health problem: is there a need for a new definition based on lipotoxicity effects? Biochem Biophys Acta 2010;1801:400-404.

2 Tan CY, Vidal-Puig A: Adipose tissue expandability: the metabolic problems of obesity may arise from inability to become more obese. Biochem Soc Trans 2008;36:935-940.

$>3$ Virtue S, Vidal-Puig A: Adipose tissue expandability, lipotoxicity and the metabolic syndrome - an allostatic perspective. Biochim Biophys Acta 2010;1801:338-349.

${ }_{4}$ Gonzales ABD, Hartge P, Cerhan JR, Flint AJ, Hannan L, MacInnis RJ, et al: Body-mass index and mortality among 1.46 millions white adults. N Engl J Med 2010;363:2211-2219.

5 The Emerging Risk Factors Collaboration: Separate and combined associations of body-mass index and abdominal adiposity with cardiovascular disease: collaborative analysis of 58 prospective studies. Lancet 2011;377:1085-1095.

6 Bigaard J, Frederiksen K, Tjønneland A, Thomsen BL, Overvad K, Heitmann BL, Sørensen TIA Body fat and fat-free mass and all-cause mortality. Obes Res 2004;12:1042-1049.

7 Stegger JG, Schmidt EB, Obel T, Berentzen TL, Tjønneland A, Sørensen TIA, Overvad K: Body composition and body fat distribution in relation to later risk of acute myocardial infarction: a Danish follow-up study. Int J Obes (Lond) 2011; doi:10.1038/ijo.2010.278

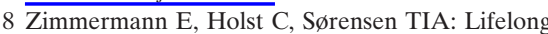
doubling of mortality in men entering adult life as obese. Int J Obes (Lond) 2011; doi:10.1038/ ijo.2010.274.

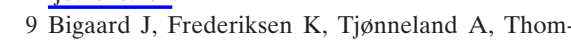
sen BL, Overvad K, Heitmann BL, Sørensen TIA: Waist circumference and body composition in relation to all-cause mortality in middle-aged men and women. Int J Obes (Lond) 2005;29:778-784.

10 Kotronen A, Yki-Järvinen H, Sevastianova K, Bergholm R, Hakkarainen A, Pietiläinen $\mathrm{KH}$, Juurinen L, Lundbom N, Sørensen TIA: Comparison of the relative contributions of intra-abdominal and liver fat to components of the metabolic syndrome. Obesity (Silver Spring). 2011;19:23-28.

-11 Jakobsen MU, Berentzen T, Sørensen TIA, Over$\operatorname{vad} \mathrm{K}$ : Abdominal obesity and fatty liver. Epidemiol Rev 2007;29:77-87.

12 Speliotes EK, Butler JL, Palmer CD, Voight BF; GIANT Consortium; MIGen Consortium; NASH CRN, Hirschhorn JN: PNPLA3 variants specifically confer increased risk for histologic nonalcoholic fatty liver disease but not metabolic disease. Hepatology 2010;52:904-912.

13 Sørensen TIA: Challenges in the study of causation of obesity. Proc Nutr Soc 2009;68:43-54.

14 Flatt JP: Issues and misconceptions about obesity. Obesity 2011;19:676-686.

15 Vidal-Puig A, Unger R: Special issue on lipotoxicity. Biochim Biophys Acta 2010;1801:207-208.

16 DeFronzo RA, Tripathy D, Schwenke DC, Barnerji MA, Bray GA, Buchanan TA, et al: Pioglitazone for diabetes prevention in impaired glucose tolerance. N Engl J Med 2011;364:11041115.

17 Berentzen T, Sørensen TIA: Effects of intended weight loss on morbidity and mortality: possible explanations of controversial results. Nutr Rev 2006; 64:502-507. 